\title{
Novel flavon stabilization with trimaximal neutrino mixing
}

\author{
So Chigusa, ${ }^{1}$ Shinta Kasuya, ${ }^{2}$ and Kazunori Nakayama ${ }^{1,3}$ \\ ${ }^{1}$ Department of Physics, Faculty of Science, The University of Tokyo, Bunkyo-ku, Tokyo 113-0033, Japan \\ ${ }^{2}$ Department of Mathematics and Physics, Kanagawa University, Kanagawa 259-1293, Japan \\ ${ }^{3}$ Kavli Institute for the Physics and Mathematics of the Universe (WPI), \\ The University of Tokyo, Kashiwa, Chiba 277-8583, Japan
}

(Received 7 June 2019; published 19 July 2019)

\begin{abstract}
We construct a supersymmetric $S_{4}$ flavor symmetry model with one of the trimaximal neutrino mixing patterns, the so-called $\mathrm{TM}_{1}$, by using the novel way to stabilize flavons, which we proposed recently. The flavons are assumed to have tachyonic supersymmetry breaking mass terms and stabilized by higherdimensional terms in the potential. We can obtain the desired alignment structure of the flavon vacuum expectation values to realize neutrino masses and mixings consistent with the current observations. This mechanism naturally avoids the appearance of dangerous cosmological domain walls. Although we study an $S_{4}$ model in this paper, our mechanism is universal and can be applied to many flavor models based on discrete flavor symmetry.
\end{abstract}

DOI: 10.1103/PhysRevD.100.015030

\section{INTRODUCTION}

Discrete flavor symmetry is often introduced in order to naturally explain the observed patterns of neutrino masses and mixings $[1,2]$. The discovery of the nonzero reactor mixing angle $\theta_{13}[3,4]$ had a great impact on the model building of the flavor symmetry and there are several ways to construct models consistent with observational data [5-8].

One of the drawbacks of such models is that the spontaneous breakdown of the discrete flavor symmetry may lead to the formation of domain walls in the early Universe, which is problematic in cosmology. Some of the discrete symmetry may be anomalous under the gauge interaction [9-18] and thus softly broken by quantum effects, but it turns out that it does not resolve the domain wall problem [19]. See also Refs. [20,21] for related works.

Recently we proposed a novel and simple way to avoid the domain wall problem in models with discrete flavor symmetry [22]. The idea is that the flavons, which are Higgs fields responsible for the spontaneous breaking of flavor symmetry, are stabilized by higher-dimensional potential balanced by tachyonic supersymmetry (SUSY) breaking mass terms. Then the flavor symmetry is already broken during inflation due to the negative Hubble-induced

Published by the American Physical Society under the terms of the Creative Commons Attribution 4.0 International license. Further distribution of this work must maintain attribution to the author(s) and the published article's title, journal citation, and DOI. Funded by SCOAP ${ }^{3}$. mass terms and never restored thereafter. ${ }^{1}$ Thus domain walls are inflated away and do not exist in the whole patch of the observable Universe. Moreover, this novel flavon stabilization mechanism significantly simplifies the field content of the flavon sector. We do not need to introduce driving fields [24] or any other additional field to obtain the flavon vacuum expectation values (VEVs) with desired alignment structure. ${ }^{2}$ An explicit construction based on the $A_{4}$ flavor symmetry was made in Ref. [22].

In this paper, we apply the general argument of Ref. [22] to the case of $S_{4}$ flavor symmetry. Models based on the $S_{4}$ flavor symmetry can lead to the so-called trimaximal neutrino mixings [28-39]. In particular, certain structure of the flavon VEVs may lead to one of the trimaximal patterns, the so-called $\mathrm{TM}_{1}$, which is consistent with current observations $[8,38]$. We explicitly show that the flavon VEV alignment that leads to the $\mathrm{TM}_{1}$ is realized along the line of Ref. [22] and the resulting neutrino masses and mixings are consistent with the recent observational data $[40,41]$. This mixing pattern $\mathrm{TM}_{1}$ will be tested in the near future by the more precise measurement of neutrino mixing angles.

In Sec. II, we briefly overview our setup of the $S_{4}$ flavor model. In Sec. III, we see that particular flavon VEV alignments lead to the so-called $\mathrm{TM}_{1}$ pattern of the neutrino mixings and it is consistent with current observations.

\footnotetext{
${ }^{1}$ In order for the flavon not to overshoot the origin of the scalar potential dynamically, it is essential to stabilize with higherdimensional potential terms $[22,23]$.

${ }^{2}$ See Refs. [25-27] for stabilization of flavons in the nonSUSY case.
} 
A novel way to obtain such a flavon VEV alignment is explained in detail in Sec. IV and we give a concrete example in Sec. V. We conclude in Sec. VI.

\section{BRIEF DESCRIPTION OF $S_{4}$ FLAVOR MODEL}

The superpotential consists of the charged lepton sector $W_{\ell}$, neutrino sector $W_{\nu}$ and flavon sector $W_{\mathrm{f}}$,

$$
W=W_{\ell}+W_{\nu}+W_{\mathrm{f}} .
$$

We focus on $W_{\ell}$ and $W_{\nu}$ in this section. The flavon part $W_{\mathrm{f}}$ is discussed in detail in Secs. IV and V where we explain how the desired flavon VEV alignments are obtained.

The superpotential of the charged lepton sector is assumed to be

$$
\begin{aligned}
W_{\ell}= & \frac{y_{\tau}}{\Lambda} \tau^{c} H_{d}\left(\phi_{\ell} \ell\right)_{\mathbf{1}}+\frac{y_{\mu}}{\Lambda^{2}} \mu^{c} H_{d}\left(\phi_{\ell}^{2} \ell\right)_{\mathbf{1}^{\prime}} \\
& +\frac{1}{\Lambda^{3}} e^{c} H_{d}\left(y_{e}^{\prime} \phi_{\ell}^{3} \ell\right)_{\mathbf{1}},
\end{aligned}
$$

where $\ell$ is the lepton doublet, $e^{c}, \mu^{c}$, and $\tau^{c}$ are respectively the right-handed electron, muon, and tau superfields, $H_{d}$ is the down-type Higgs doublet, $\phi_{\ell}$ is the flavon field in the charged lepton sector, $\Lambda$ denotes the cutoff scale and $y_{e}^{\prime}, y_{\mu}$, and $y_{\tau}$ are coupling constants. Charge assignments under the $S_{4}$ flavor symmetry are summarized in Table I. See the Appendix A for our convention and notation of the $S_{4}$ group representations and $S_{4}$ products. There are three possible contractions in the third term of (2), but all of them lead to the same structure of the mass matrix in the argument below. After taking the VEV of

$$
\left\langle\phi_{\ell}\right\rangle=\left(0, v_{\ell}, 0\right)^{T},
$$

we obtain the diagonal charged lepton mass matrix as

$$
\mathcal{M}_{\ell}=\frac{v_{\ell} v_{d}}{\Lambda}\left(\begin{array}{ccc}
y_{e} v_{\ell}^{2} / \Lambda^{2} & 0 & 0 \\
0 & 2 y_{\mu} v_{\ell} / \Lambda & 0 \\
0 & 0 & y_{\tau}
\end{array}\right) \text {, }
$$

with $v_{d}$ being the VEV of the down-type Higgs. Note that $y_{e}$ in this expression is some linear combination of $y_{e}^{\prime} \mathrm{s}$ defined for each contraction of the third term in (2). One can take charged lepton masses real and positive without loss of generality. The mass hierarchy of the charged leptons

TABLE I. Charge assignments under $S_{4}, R$-symmetry $\mathrm{U}(1)_{R}$, and $Z_{6}^{\ell}$ for leptons and various Higgs and flavon fields.

\begin{tabular}{lccccccccccc}
\hline \hline & $\ell$ & $e^{c}$ & $\mu^{c}$ & $\tau^{c}$ & $H_{u}$ & $H_{d}$ & $\phi_{\ell}$ & $\phi_{\mathbf{1}}$ & $\phi_{\mathbf{2}}$ & $\phi_{\mathbf{3}^{\prime}}$ & $\psi_{\mathbf{3}^{\prime}}$ \\
\hline$S_{4}$ & $\mathbf{3}$ & $\mathbf{1}$ & $\mathbf{1}^{\prime}$ & $\mathbf{1}$ & $\mathbf{1}$ & $\mathbf{1}$ & $\mathbf{3}$ & $\mathbf{1}$ & $\mathbf{2}$ & $\mathbf{3}^{\prime}$ & $\mathbf{3}^{\prime}$ \\
$\mathrm{U}(1)_{R}$ & $5 / 6$ & $1 / 6$ & $1 / 2$ & $5 / 6$ & 0 & 0 & $1 / 3$ & $1 / 3$ & $1 / 3$ & $1 / 3$ & $1 / 3$ \\
$Z_{6}^{\ell}$ & 0 & -3 & -2 & -1 & 0 & 0 & 1 & 0 & 0 & 0 & 0 \\
\hline \hline
\end{tabular}

may be explained for $v_{\ell} / \Lambda \sim \mathcal{O}(0.1)$. Since the charged lepton mass matrix is already diagonal, we only have to consider the structure of the neutrino mass matrix when discussing the lepton mixings in the weak interaction.

The superpotential of the neutrino sector is written as

$$
\begin{aligned}
W_{\nu}= & \frac{H_{u}^{2}}{\Lambda^{2}}\left[c_{1} \phi_{\mathbf{1}}(\ell \ell)_{\mathbf{1}}+c_{2} \phi_{\mathbf{2}}(\ell \ell)_{\mathbf{2}}+c_{3^{\prime}} \phi_{\mathbf{3}^{\prime}}(\ell \ell)_{\mathbf{3}^{\prime}}\right. \\
& \left.+c_{\psi} \psi_{\mathbf{3}^{\prime}}(\ell \ell)_{\mathbf{3}^{\prime}}\right],
\end{aligned}
$$

where $H_{u}$ denotes the up-type Higgs doublet. Here we have an $S_{4}$-singlet $\phi_{1}$, an $S_{4}$-doublet $\phi_{2}$, and two $S_{4}$-triplets $\phi_{3^{\prime}}$ and $\psi_{3^{\prime}}$. Note that the coupling of $\phi_{\ell}$ to the neutrino sector is forbidden by the $Z_{6}^{\ell}$ symmetry whose charge assignments are given in Table I. These flavons are assumed to develop VEVs of the form

$$
\begin{aligned}
& \left\langle\phi_{1}\right\rangle=v_{1}, \quad\left\langle\phi_{2}\right\rangle=v_{2}\left(\begin{array}{l}
1 \\
1
\end{array}\right), \quad\left\langle\phi_{3^{\prime}}\right\rangle=v_{3^{\prime}}\left(\begin{array}{l}
1 \\
1 \\
1
\end{array}\right), \\
& \left\langle\psi_{\mathbf{3}^{\prime}}\right\rangle=v_{\psi}\left(\begin{array}{c}
0 \\
1 \\
-1
\end{array}\right) .
\end{aligned}
$$

Then the neutrino mass matrix is given by

$$
\begin{aligned}
\mathcal{M}_{\nu}= & \frac{v_{u}^{2}}{\Lambda^{2}}\left[w_{1}\left(\begin{array}{ccc}
1 & 0 & 0 \\
0 & 0 & 1 \\
0 & 1 & 0
\end{array}\right)+w_{2}\left(\begin{array}{ccc}
0 & 1 & 1 \\
1 & 1 & 0 \\
1 & 0 & 1
\end{array}\right)\right. \\
& \left.+w_{3^{\prime}}\left(\begin{array}{ccc}
2 & -1 & -1 \\
-1 & 2 & -1 \\
-1 & -1 & 2
\end{array}\right)+w_{\psi}\left(\begin{array}{ccc}
0 & 1 & -1 \\
1 & 2 & 0 \\
-1 & 0 & -2
\end{array}\right)\right],
\end{aligned}
$$

where $w_{1} \equiv c_{1} v_{1}, w_{2} \equiv c_{2} v_{2}, w_{3^{\prime}} \equiv c_{3^{\prime}} v_{3^{\prime}}$, and $w_{\psi} \equiv c_{\psi} v_{\psi}$. Here $v_{u}$ is the VEV of the up-type Higgs. For $v_{\psi}=0$, it becomes the neutrino mass matrix that is diagonalized by the tribimaximal mixing matrix $[42,43]$

$$
U_{\mathrm{TB}}=\left(\begin{array}{ccc}
2 / \sqrt{6} & 1 / \sqrt{3} & 0 \\
-1 / \sqrt{6} & 1 / \sqrt{3} & 1 / \sqrt{2} \\
-1 / \sqrt{6} & 1 / \sqrt{3} & -1 / \sqrt{2}
\end{array}\right) \text {, }
$$

and the neutrino mass eigenvalues are given by

$$
m_{\nu}=\frac{v_{u}^{2}}{\Lambda^{2}}\left(w_{1}-w_{2}+3 w_{3^{\prime}}, w_{1}+2 w_{2},-w_{1}+w_{2}+3 w_{3^{\prime}}\right)
$$


The tribimaximal mixing, however, is already ruled out by experiments after the observation of nonzero $\theta_{13}$. In addition, the recent observation favors nonzero Dirac $C P$ phase $\delta .^{3}$

The nonzero $v_{\psi}$ breaks the tribimaximal symmetry but still there remains a $Z_{2}$ symmetry generated by the combination $S U$ of the $S_{4}$ group elements. It leads to the trimaximal mixing (especially, the so-called $\mathrm{TM}_{1}$ ), which can fit the experimental results well [8]. It is convenient to divide the full mixing matrix $U^{\nu}$ into the tribimaximal part and the additional 2-3 rotation part,

$$
U^{\nu}=U_{\mathrm{TB}} U_{23}, \quad U_{23} \equiv\left(\begin{array}{cc}
1 & 0 \\
0 & u_{23}
\end{array}\right)
$$

where $u_{23}$ is a $2 \times 2$ unitary matrix. Then we find

$$
U^{\nu T} \mathcal{M}_{\nu} U^{\nu}=\frac{v_{u}^{2}}{\Lambda^{2}}\left(\begin{array}{cc}
w_{1}-w_{2}+3 w_{3^{\prime}} & 0 \\
0 & u_{23}^{T}\left(\begin{array}{cc}
w_{1}+2 w_{2} & \sqrt{6} w_{\psi} \\
\sqrt{6} w_{\psi} & -w_{1}+w_{2}+3 w_{3^{\prime}}
\end{array}\right) u_{23}
\end{array}\right)
$$

By choosing the unitary matrix $u_{23}$ appropriately, this can be diagonalized and we obtain the full mixing matrix $U^{\nu}$ from which we can deduce the various neutrino mixing angles and $C P$ phases. A concrete procedure is discussed in the next section.

Assuming that all $w_{1}, w_{2}, w_{3^{\prime}}$, and $w_{\psi}$ are the same order of magnitude and denoting their typical values by $w$, we obtain the neutrino mass scale as

$$
m_{\nu} \sim 0.3 \mathrm{eV}\left(\frac{10^{12} \mathrm{GeV}}{\Lambda}\right)^{2}\left(\frac{w}{10^{10} \mathrm{GeV}}\right) \sin ^{2} \beta
$$

where $\tan \beta \equiv v_{u} / v_{d}$. The typical value of the flavon VEV $w$ crucially depends on the stabilization mechanism. In our model presented in Sec. V, it is given by $w \sim\left(m_{\text {soft }} \Lambda^{3}\right)^{1 / 4}$, where $m_{\text {soft }}$ is the soft SUSY breaking mass. Then we can reproduce the observed neutrino mass differences with some amount of tuning of $w_{1}, w_{2}, w_{3^{\prime}}$, and $w_{\psi}$.

\section{NEUTRINO MIXING IN TM}

Let us describe how to diagonalize (11) by choosing $u_{23}$. A general $2 \times 2$ unitary matrix may be parametrized as

$$
U^{\nu}=\left(\begin{array}{ccc}
2 / \sqrt{6} & \cos \theta / \sqrt{3} & e^{i \eta} \sin \theta / \sqrt{3} \\
-1 / \sqrt{6} & \cos \theta / \sqrt{3}-e^{-i \eta} \sin \theta / \sqrt{2} & \cos \theta / \sqrt{2}+e^{i \eta} \sin \theta / \sqrt{3} \\
-1 / \sqrt{6} & \cos \theta / \sqrt{3}+e^{-i \eta} \sin \theta / \sqrt{2} & -\cos \theta / \sqrt{2}+e^{i \eta} \sin \theta / \sqrt{3}
\end{array}\right)\left(\begin{array}{ccc}
1 & 0 & 0 \\
0 & e^{i \alpha} & 0 \\
0 & 0 & e^{i \beta}
\end{array}\right) .
$$

On the other hand, the mixing matrix (MNS matrix) is usually parametrized as [44]

$$
U^{\mathrm{MNS}}=\left(\begin{array}{ccc}
c_{12} c_{13} & s_{12} c_{13} & s_{13} e^{-i \delta} \\
-s_{12} c_{23}-c_{12} s_{23} s_{13} e^{i \delta} & c_{12} c_{23}-s_{12} s_{23} s_{13} e^{i \delta} & s_{23} c_{13} \\
s_{12} s_{23}-c_{12} c_{23} s_{13} e^{i \delta} & -c_{12} s_{23}-s_{12} c_{23} s_{13} e^{i \delta} & c_{23} c_{13}
\end{array}\right)\left(\begin{array}{ccc}
1 & 0 & 0 \\
0 & e^{i \alpha_{21} / 2} & 0 \\
0 & 0 & e^{i \alpha_{31} / 2}
\end{array}\right),
$$

\footnotetext{
${ }^{3}$ Neutrino mass eigenvalues obtained here are complex in general. One of them, $m_{\nu_{1}}$, for example, can be made real by the common phase rotation of $\left(\nu_{1}, \nu_{2}, \nu_{3}\right)$, which is accompanied by the opposite common phase rotation of $(e, \mu, \tau)$ to keep the mixing matrix intact. Thus there remain two physical Majorana phases. We always take such a basis in the following.
} 
where $c_{i j}=\cos \theta_{i j}, s_{i j}=\sin \theta_{i j}, \delta$ is the Dirac $C P$ phase, and $\alpha_{21}$ and $\alpha_{31}$ are Majorana $C P$ phases. Here we take the basis in which the neutrino mass matrix is real and diagonal.

The mixing matrix $U^{\nu}$ can be reduced to the form of $U^{\mathrm{MNS}}$ (16) by using the freedom to rotate the phase of charged leptons and neutrinos. Even without performing such a concrete phase rotation, one can conveniently deduce the mixing parameters and the Dirac $C P$ phase by using the rotation invariant quantity as

$s_{13}^{2}=\left|U_{e 3}^{\nu}\right|^{2}, \quad s_{12}^{2}=\frac{\left|U_{e 2}^{\nu}\right|^{2}}{1-\left|U_{e 3}^{\nu}\right|^{2}}, \quad s_{23}^{2}=\frac{\left|U_{\mu 3}^{\nu}\right|^{2}}{1-\left|U_{e 3}^{\nu}\right|^{2}}$,

and

$J_{\mathrm{CP}} \equiv \operatorname{Im}\left(U_{\mu 3}^{\nu} U_{e 3}^{\nu *} U_{e 2}^{\nu} U_{\mu 2}^{\nu *}\right)=s_{12} s_{23} s_{13} c_{12} c_{23} c_{13}^{2} \sin \delta$.

We can also derive sum rules independent of $\theta$ and $\eta$. One of them is obtained from $\left|U_{e 1}^{\nu}\right|^{2}=\left(c_{12} c_{13}\right)^{2}$ as

$$
c_{12} c_{13}=\frac{2}{\sqrt{6}} \simeq 0.816
$$

which fits well with the observation $c_{12}^{\text {(obs) }} c_{13}^{\text {(obs) }}=$ $0.821_{-0.008}^{+0.007}$ within $1 \sigma$ [40]. Another one is extracted from comparing $\left|U_{\mu 1}^{\nu}\right|^{2}$ and $\left|U_{\tau 1}^{\nu}\right|^{2}$ with the MNS parametrization,

$$
\begin{aligned}
& \cos \left(2 \theta_{23}\right)\left(\frac{2}{3}-\cos \left(2 \theta_{12}\right)\right)+\sin \left(2 \theta_{12}\right) \sin \left(2 \theta_{23}\right) \\
& \quad \times \sin \theta_{13} \cos \delta=0 .
\end{aligned}
$$

Substituting the best-fit values $\theta_{23}^{(\mathrm{obs})}=49.6^{\circ}, \theta_{12}^{(\mathrm{obs})}=$ $33.82^{\circ}$, and $\theta_{13}^{(\mathrm{obs})}=8.61^{\circ}$ [40], we obtain $\cos \delta \simeq 0.33$. $^{4}$ Note that there are no more nontrivial sum rules in the present model. We have four complex model parameters $w_{1}, w_{2}, w_{3^{\prime}}$, and $w_{\psi}$ to construct neutrino mass matrix, but an overall phase is irrelevant and hence we have seven real parameters (two of which are parametrized by $\theta$ and $\eta$ ). The number of the physical observables is 9 , consisting of three neutrino mass eigenvalues, three mixing angles, one

\footnotetext{
${ }^{4}$ If one assumes that the deviation from the tribimaximal form is small, one may regard $\theta_{13}$ as a small parameter and expand as $\theta_{23}=\pi / 4+\Delta \theta_{23}$. Note that the deviation of $\theta_{12}$ from its tribimaximal value $\left(c_{12}=\sqrt{2 / 3}\right)$ is second order in terms of $\theta_{13}$ because of the constraint (19). Then we have
}

$$
\Delta \theta_{23} \simeq \sqrt{2} \theta_{13} \cos \delta .
$$

Dirac $C P$ phase, and two Majorana $C P$ phases. Thus there should be two nontrivial sum rules in the physical observables.

Figure 1 shows the observationally preferred region of $\sin ^{2} \theta_{13}$ and $\sin ^{2} \theta_{12}$ (top), and $\sin ^{2} \theta_{23}$ and $\delta$ (bottom) within $1 \sigma, 2 \sigma$, and $3 \sigma$ ranges [40,41]. A line in the top panel indicates the sum rule (19), a prediction of $\mathrm{TM}_{1}$. This is solely determined by the model parameter $\theta$ and independent of $\eta$. It is seen that the line crosses the observationally preferred region and hence $\theta$ is constrained to some narrow range. Once we fix $\theta$, we can draw a line on the plane of $\sin ^{2} \theta_{23}$ and $\delta$ by changing $\eta$. Three lines in the bottom panel indicate the predictions of $\mathrm{TM}_{1}$ for typical values of $\theta$ that reproduce the observed values of $\theta_{12}$ and $\theta_{13}$. We can see that the $\mathrm{TM}_{1}$ prediction is consistent with current experimental results. If future observations determine $\delta$ and $\theta_{23}$ more precisely, the $\mathrm{TM}_{1}$ can be tested more crucially.
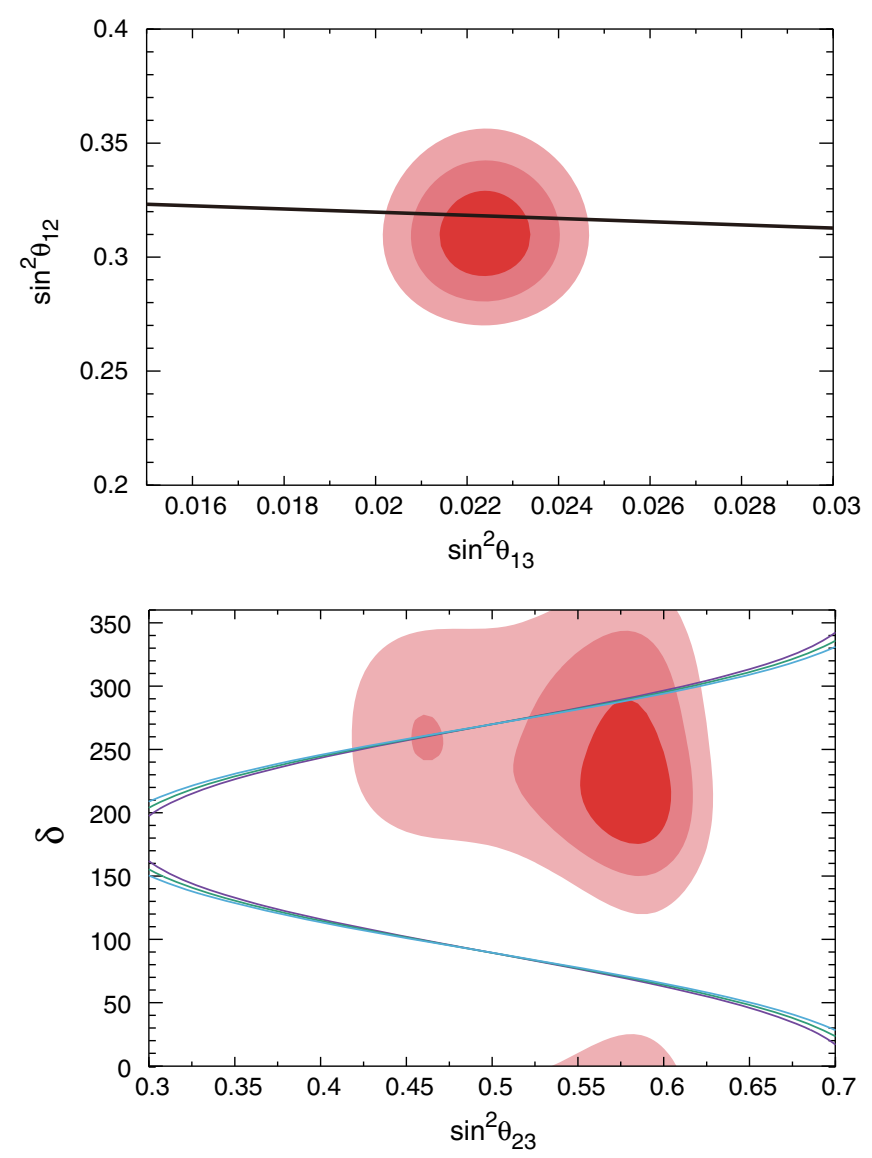

FIG. 1. (Top) Observationally preferred region of $\sin ^{2} \theta_{13}$ and $\sin ^{2} \theta_{12}$ within $1 \sigma, 2 \sigma$, and $3 \sigma$ ranges [40,41]. A line indicates the sum rule (19), a prediction of $\mathrm{TM}_{1}$. (Bottom) Observationally preferred region of $\sin ^{2} \theta_{23}$ and $\delta$ within $1 \sigma, 2 \sigma$, and $3 \sigma$ ranges $[40,41]$. Three lines indicate the predictions of $\mathrm{TM}_{1}$ for typical values of $\theta$ that reproduce the observed values of $\theta_{12}$ and $\theta_{13}$. 


\section{NOVEL FLAVON STABILIZATION: GENERAL ARGUMENT}

Let us adopt the idea of Ref. [22] to obtain the desired alignment structure of the flavon VEVs in the $S_{4}$ flavor model introduced in the previous sections. In this section, we describe some general arguments about our method for the flavon stabilization, while we give a concrete example of the superpotential for the flavon sector in the next section.

The basic idea is that the flavon fields are stabilized by the balance between the negative soft SUSY breaking mass and nonrenormalizable terms in the potential. The latter comes from nonrenormalizable superpotentials. In our setup, as shown in Table I, flavons have $\mathrm{U}(1)_{R}$ charge of $1 / 3$ and hence only terms with the sixth power of flavons are allowed in the superpotential. Schematically, we have

$$
W_{\mathrm{f}} \sim \frac{\phi^{6}}{\Lambda^{3}},
$$

where the flavon fields are collectively denoted as $\phi$. This is a shorthand notation and there are actually many ways of contracting $S_{4}$-charged flavons. Together with the SUSY breaking mass term, the scalar potential has the form as

$$
V \sim-m^{2}|\phi|^{2}+\frac{|\phi|^{10}}{\Lambda^{6}},
$$

and the flavon fields obtain VEVs of $\langle|\phi|\rangle \sim\left(m \Lambda^{3}\right)^{1 / 4}$. What is nontrivial is whether or not we can obtain the alignment structure given in (3) and (6). Here we show that the configuration (3) and (6) is always an extremum of the scalar potential independent of the detailed form of the superpotential under some assumptions.

First let us consider $\phi_{\ell}$. Because of the $Z_{6}^{\ell}$ symmetry, it is not mixed with other flavons in the superpotential. Therefore, in terms of components $\phi_{\ell}=\left(\phi_{\ell, 1}, \phi_{\ell, 2}, \phi_{\ell, 3}\right)^{T}$, the superpotential may be generically written as

$$
W_{\mathrm{f}, \ell}=\frac{1}{\Lambda^{3}}\left(\phi_{\ell, 2}^{6}+\phi_{\ell, 2}^{5} \phi_{\ell, 1}+\phi_{\ell, 2}^{5} \phi_{\ell, 3}+\cdots\right),
$$

where dots represent terms with higher powers of $\phi_{\ell, 1}$ or $\phi_{\ell, 3}$ and we omitted $\mathcal{O}(1)$ numerical coefficients for simplicity. First, by substituting $\phi_{\ell}=\left(0, \phi_{\ell, 2}, 0\right)^{T}$ one can minimize the potential along the $\phi_{\ell, 2}$ direction to find $\operatorname{VEV}\left\langle\left|\phi_{\ell, 2}\right|\right\rangle=v_{\ell}$. A necessary and sufficient condition for this configuration to be an extremum of the potential is that there are no linear terms with respect to $\phi_{\ell, 1}$ and $\phi_{\ell, 3}$ in the superpotential when expanded around the configuration. ${ }^{5}$ Actually, we can show that such terms are forbidden by

\footnotetext{
${ }^{5}$ Here linear terms with respect to $\phi_{\ell, i}$ means terms of the form of $v^{5} \phi_{\ell, i} / \Lambda^{3}$, where $v$ collectively represents the nonzero VEV of various flavons. We use the same terminology in the following discussion.
}

symmetry. In our setup, the flavon superpotential must be invariant under $S_{4}$ symmetry and also $Z_{6}^{\ell}$ symmetry under which the flavon is rotated like $\phi_{\ell} \rightarrow \Omega \phi_{\ell}$ with $\Omega \equiv e^{2 \pi i / 6}$. Then, for example, the superpotential must be invariant under the transformation

$$
\phi_{\ell} \rightarrow \Omega^{2} T \phi_{\ell}=\left(\begin{array}{ccc}
\Omega^{2} & 0 & 0 \\
0 & 1 & 0 \\
0 & 0 & \Omega^{4}
\end{array}\right) \phi_{\ell},
$$

where $T$ is an element of three-dimensional representation of $S_{4}$ given in (A2). Under this transformation, $\phi_{\ell, 2}$ is invariant but $\phi_{\ell, 1}$ and $\phi_{\ell, 3}$ are not. Therefore, we cannot have terms linear in $\phi_{\ell, 1}$ and $\phi_{\ell, 3}$ in the superpotential and the configuration (3) is indeed an extremum of the potential. Note that the combination $\Omega^{2} T$ used here is the generator of the remaining $Z_{3} \subset S_{4} \times Z_{6}^{\ell}$ symmetry that is retained in $W_{\mathrm{f}, \ell}$ even after $\phi_{\ell}$ takes the nonzero VEV of (3). In order to show that it is a minimum of the potential, one must check terms quadratic in $\phi_{\ell, 1}$ and $\phi_{\ell, 3}$, which depend on the detailed forms of the superpotential. In the next section, we give a concrete example in which the configuration (3) is actually a minimum of the potential.

Next, flavons $\phi_{1}, \phi_{2}, \phi_{3^{\prime}}$, and $\psi_{3^{\prime}}$ must have the same quantum numbers (except for the $S_{4}$ charge) due to the coupling (5) and hence they are in general mixed with each other in the flavon superpotential. What is dangerous is the mixing between $\phi_{3^{\prime}}$ and $\psi_{3^{\prime}}$ that would spoil the vacuum alignment (6). In order to forbid dangerous mixings between $\phi_{3^{\prime}}$ and $\psi_{3^{\prime}}$, we assign different charges to them under some symmetry. For this purpose, one can modify the superpotential of the neutrino sector (5) as follows [38]:

$$
\begin{aligned}
W_{\nu}= & \frac{H_{u}^{2}}{\Lambda^{3}}\left[\xi_{\mathbf{1}}\left(c_{1} \phi_{\mathbf{1}}(\ell \ell)_{\mathbf{1}}+c_{2} \phi_{\mathbf{2}}(\ell \ell)_{\mathbf{2}}+c_{3^{\prime}} \phi_{\mathbf{3}^{\prime}}(\ell \ell)_{\mathbf{3}^{\prime}}\right)\right. \\
& \left.+c_{\psi} \xi_{\mathbf{1}^{\prime}} \psi_{\mathbf{3}^{\prime}}(\ell \ell)_{\mathbf{3}^{\prime}}\right]
\end{aligned}
$$

where we introduced additional $S_{4}$ singlet flavons $\xi_{1}$ and $\xi_{1}^{\prime}$ and imposed additional $Z_{6}^{\xi}, Z_{6}^{\xi^{\prime}}$, and $Z_{12}^{\xi \xi^{\prime}}$ symmetry as presented in Table II. Note that $\mathrm{U}(1)_{R}$ charge assignments are modified from those of Table I. These additional flavons are assumed to have VEVs of $\left\langle\xi_{1}\right\rangle=v_{\xi}$ and $\left\langle\xi_{1}^{\prime}\right\rangle=v_{\xi^{\prime}}$. The structure of neutrino masses and mixings described in Secs. II and III is unchanged after reinterpreting $w_{1}=c_{1} v_{\xi} v_{1} / \Lambda$, and so on. The additional $Z_{6}^{\xi}, Z_{6}^{\xi^{\prime}}$, and $Z_{12}^{\xi \xi^{\prime}}$ symmetry, combined with $\mathrm{U}(1)_{R}$ symmetry, restricts the form of the flavon superpotential to be

$$
\begin{aligned}
W_{\mathrm{f}}= & W_{\mathrm{f}, \ell}\left(\phi_{\ell}\right)+W_{\mathrm{f}, \phi}\left(\phi_{\mathbf{1}}, \phi_{\mathbf{2}}, \phi_{\mathbf{3}^{\prime}}\right)+W_{\mathrm{f}, \xi}\left(\xi_{\mathbf{1}}\right) \\
& +W_{\mathrm{f}, \psi}\left(\psi_{\mathbf{3}^{\prime}}\right)+W_{\mathrm{f}, \xi^{\prime}}\left(\xi_{\mathbf{1}}^{\prime}\right) .
\end{aligned}
$$

It is easy to see that $\phi_{1}, \xi_{1}$, and $\xi_{1}^{\prime}$ are stabilized by using terms like $\phi^{6} / \Lambda^{3}$, where $\phi$ collectively denotes a $S_{4}$ 
TABLE II. Charge assignments under $S_{4}, R$-symmetry $\mathrm{U}(1)_{R}, Z_{6}^{\ell}, Z_{6}^{\xi}, Z_{6}^{\xi^{\prime}}$, and $Z_{12}^{\xi \xi^{\prime}}$ for leptons, Higgs and flavon fields.

\begin{tabular}{|c|c|c|c|c|c|c|c|c|c|c|c|c|c|}
\hline & $\ell$ & $e^{c}$ & $\mu^{c}$ & $\tau^{c}$ & $H_{u}$ & $H_{d}$ & $\phi_{\ell}$ & $\phi_{1}$ & $\phi_{2}$ & $\phi_{\mathbf{3}^{\prime}}$ & $\psi_{\mathbf{3}^{\prime}}$ & $\xi_{1}$ & $\xi_{1}^{\prime}$ \\
\hline$S_{4}$ & 3 & 1 & $\mathbf{1}^{\prime}$ & 1 & 1 & 1 & 3 & 1 & 2 & $\mathbf{3}^{\prime}$ & $\mathbf{3}^{\prime}$ & 1 & 1 \\
\hline $\mathrm{U}(1)_{R}$ & $2 / 3$ & $1 / 3$ & $2 / 3$ & 1 & 0 & 0 & $1 / 3$ & $1 / 3$ & $1 / 3$ & $1 / 3$ & $1 / 3$ & $1 / 3$ & $1 / 3$ \\
\hline$Z_{6}^{\ell}$ & 0 & -3 & -2 & -1 & 0 & 0 & 1 & 0 & 0 & 0 & 0 & 0 & 0 \\
\hline$Z_{6}^{\xi}$ & 0 & 0 & 0 & 0 & 0 & 0 & 0 & -1 & -1 & -1 & 0 & 1 & 0 \\
\hline$Z_{6}^{\xi^{\prime}}$ & 0 & 0 & 0 & 0 & 0 & 0 & 0 & 0 & 0 & 0 & -1 & 0 & 1 \\
\hline$Z_{12}^{\xi \xi^{\prime}}$ & 5 & -5 & -5 & -5 & 0 & 0 & 0 & 0 & 0 & 0 & 0 & 2 & 2 \\
\hline
\end{tabular}

singlet flavon field. Thus we only need to focus on $\phi_{2}, \phi_{3^{\prime}}$, and $\psi_{\mathbf{3}^{\prime}}$ below.

For $\psi_{3^{\prime}}$, it is convenient to work with the basis in which $\bar{\psi}_{3^{\prime}}=V_{\psi} \psi_{3^{\prime}}$, where

$$
V_{\psi}=\left(\begin{array}{ccc}
1 & 0 & 0 \\
0 & 1 / \sqrt{2} & -1 / \sqrt{2} \\
0 & 1 / \sqrt{2} & 1 / \sqrt{2}
\end{array}\right) .
$$

In this basis, the alignment structure (6) becomes $\left\langle\bar{\psi}_{3^{\prime}}\right\rangle=$ $\sqrt{2} v_{\psi}(0,1,0)^{T}$. By noting that $\bar{\psi}_{\mathbf{3}^{\prime}}$ transforms as $\bar{\psi}_{\mathbf{3}^{\prime}} \rightarrow$ $V_{\psi} X V_{\psi}^{-1} \bar{\psi}_{3^{\prime}}$ under any $S_{4}$ group element $X$, one finds that $\bar{\psi}_{3^{\prime}, 2}$ is invariant but $\bar{\psi}_{3^{\prime}, 1}$ and $\bar{\psi}_{3^{\prime}, 3}$ are not under the transformation

$$
\Omega^{3} V_{\psi} U V_{\psi}^{-1}=\left(\begin{array}{ccc}
-1 & 0 & 0 \\
0 & 1 & 0 \\
0 & 0 & -1
\end{array}\right) .
$$

Here $\Omega^{3}$ should be regarded as an element of $Z_{6}^{\xi^{\prime}}$ rotation. Thus we cannot have terms linear in $\bar{\psi}_{3^{\prime}, 1}$ and $\bar{\psi}_{3^{\prime}, 3}$ and hence the $\psi_{\mathbf{3}^{\prime}}$ configuration in (6) is always an extremum of the potential. In this case, $\Omega^{3} S$ and $\Omega^{3} U$ are the generators of the $Z_{2} \times Z_{2} \subset S_{4} \times Z_{6}^{\xi^{\prime}}$ subgroup that is unbroken in $W_{\mathrm{f}, \psi}$ when the VEV of $\psi_{3^{\prime}}$ in (6) is taken into account. In addition, since $\xi_{1}{ }^{\prime}$ also acquires the nonzero $\mathrm{VEV}$, the symmetry in the neutrino sector is further broken to the $Z_{2}$ subgroup generated by $\left(\Omega^{3} S\right)\left(\Omega^{3} U\right)=S U$, which is responsible for the $\mathrm{TM}_{1}$ mixing patterns.

Similar arguments hold for the other flavons, $\phi_{2}$ and $\phi_{3^{\prime}}$. In general, they are mixed in the superpotential $W_{\mathrm{f}, \phi}\left(\phi_{1}\right.$, $\left.\phi_{2}, \phi_{3^{\prime}}\right)$. Let us go to the basis in which $\bar{\phi}_{2}=V_{2} \phi_{2}$ and $\bar{\phi}_{3^{\prime}}=V_{\phi} \phi_{3^{\prime}}$, where

$$
\begin{aligned}
V_{2} & =\frac{1}{\sqrt{2}}\left(\begin{array}{cc}
1 & 1 \\
-1 & 1
\end{array}\right), \\
V_{\phi} & =\left(\begin{array}{ccc}
2 / \sqrt{6} & -1 / \sqrt{6} & -1 / \sqrt{6} \\
1 / \sqrt{3} & 1 / \sqrt{3} & 1 / \sqrt{3} \\
0 & 1 / \sqrt{2} & -1 / \sqrt{2}
\end{array}\right) .
\end{aligned}
$$

In this basis, the alignment structure (6) becomes

$$
\left\langle\bar{\phi}_{\mathbf{2}}\right\rangle=\sqrt{2} v_{\mathbf{2}}\left(\begin{array}{l}
1 \\
0
\end{array}\right), \quad\left\langle\bar{\phi}_{\mathbf{3}^{\prime}}\right\rangle=\sqrt{3} v_{\mathbf{3}^{\prime}}\left(\begin{array}{l}
0 \\
1 \\
0
\end{array}\right) .
$$

Let us note that

$$
V_{2} S V_{2}^{-1}=\left(\begin{array}{ll}
1 & 0 \\
0 & 1
\end{array}\right), \quad V_{2} S U V_{2}^{-1}=\left(\begin{array}{cc}
1 & 0 \\
0 & -1
\end{array}\right),
$$

and

$$
\begin{aligned}
V_{\phi} S V_{\phi}^{-1} & =\left(\begin{array}{ccc}
-1 & 0 & 0 \\
0 & 1 & 0 \\
0 & 0 & -1
\end{array}\right), \\
V_{\phi} S U V_{\phi}^{-1} & =\left(\begin{array}{ccc}
-1 & 0 & 0 \\
0 & 1 & 0 \\
0 & 0 & 1
\end{array}\right) .
\end{aligned}
$$

Therefore, from the invariance of the superpotential under the $S U$ transformation, terms linear in $\bar{\phi}_{2,2}$ are forbidden. Likewise, from the invariance under the $S$ transformation, we cannot have terms linear in $\bar{\phi}_{3^{\prime}, 1}$ and $\bar{\phi}_{3^{\prime}, 3}$. Therefore, for general superpotential $W_{\mathrm{f}, \phi}\left(\phi_{1}, \phi_{2}, \phi_{3^{\prime}}\right)$, we show that the configuration given in (6) is always an extremum of the potential. Again note that $S$ and $U$ are the generators of the remaining $Z_{2} \times Z_{2} \subset S_{4} \times Z_{6}^{\xi}$ symmetry of $W_{\mathrm{f}, \phi}+W_{\mathrm{f}, \xi}$ (which of course contains $S U$ ) when $\phi_{1}, \phi_{2}, \phi_{3^{\prime}}$, and $\xi$ obtain nonzero VEVs. In order to show that it is in fact a minimum of the potential, one must check the curvature around it. It depends on the detailed form of the superpotential. We give a concrete example in which the configuration (3) and (6) is actually a minimum of the potential.

\section{NOVEL FLAVON STABILIZATION: CONCRETE EXAMPLE}

We have shown that the flavon configuration given by (3) and (6) is always an extremum of the potential by just 
using the general symmetry argument in the previous section. Now we show that this configuration can actually be a minimum of the potential. As a concrete example, let us consider a simple nonrenormalizable superpotential of the flavon sector given as

$$
W_{\mathrm{f}}=W_{\mathrm{f}, \ell}+W_{\mathrm{f}, \mathbf{1}}+W_{\mathrm{f}, \mathbf{2}}+W_{\mathrm{f}, \mathbf{3}^{\prime}}^{\phi}+W_{\mathrm{f}, \boldsymbol{\xi}}+W_{\mathrm{f}, \mathbf{3}^{\prime}}^{\psi}+W_{\mathrm{f}, \xi^{\prime}},
$$

$$
\begin{gathered}
W_{\mathrm{f}, \ell}=\frac{1}{\Lambda^{3}}\left[h_{1}\left(\phi_{\ell}^{3}\right)_{\mathbf{1}^{\prime}}^{2}+h_{2}\left(\phi_{\ell}^{2}\right)_{\mathbf{1}}\left(\left(\phi_{\ell}^{2}\right)_{\mathbf{3}^{\prime}}^{2}\right)_{\mathbf{1}}\right], \\
W_{\mathrm{f}, \mathbf{1}}=\frac{g_{0}}{\Lambda^{3}} \phi_{\mathbf{1}}^{6} ; \quad W_{\mathrm{f}, \boldsymbol{\xi}}=\frac{g_{\xi}}{\Lambda^{3}} \xi_{1}^{6} ; \quad W_{\mathrm{f}, \xi^{\prime}}=\frac{g_{\xi^{\prime}}}{\Lambda^{3}} \xi_{\mathbf{1}}^{6}, \\
W_{\mathrm{f}, \mathbf{2}}=\frac{1}{\Lambda^{3}}\left[g_{1}\left(\phi_{2}^{2}\right)_{1}^{3}+g_{2}\left(\phi_{2}^{3}\right)_{\mathbf{1}}^{2}\right], \\
W_{\mathrm{f}, \mathbf{3}^{\prime}}^{\phi}=\frac{1}{\Lambda^{3}}\left[g_{3}\left(\phi_{\mathbf{3}^{\prime}}^{2}\right)_{\mathbf{1}}^{3}+g_{4}\left(\left[\phi_{\mathbf{3}^{\prime}}^{5}\right] \phi_{\mathbf{3}^{\prime}}\right)_{\mathbf{1}}\right], \\
W_{\mathrm{f}, \mathbf{3}^{\prime}}^{\psi}=\frac{1}{\Lambda^{3}}\left[g_{5}\left(\psi_{\mathbf{3}^{\prime}}^{2}\right)_{\mathbf{1}}^{3}+g_{6}\left(\left[\psi_{\mathbf{3}^{\prime}}^{5}\right] \psi_{\mathbf{3}^{\prime}}\right)_{\mathbf{1}}\right],
\end{gathered}
$$

where the block parenthesis for $\phi_{3^{\prime}}$ is recursively defined as

$$
\begin{gathered}
{\left[\phi_{3^{\prime}}\right] \equiv \phi_{3^{\prime}},} \\
{\left[\phi_{3^{\prime}}^{n}\right] \equiv\left(\left[\phi_{3^{\prime}}^{n-1}\right] \phi_{3^{\prime}}\right)_{3^{\prime}},}
\end{gathered}
$$

and the same applies to $\psi_{3^{\prime}}$. The above definition uniquely specifies the way of contraction in each term. Some comments are in order. First, we assume that the superpotential of each flavon is separated as $W_{\mathrm{f}, \phi}=W_{\mathrm{f}, \mathbf{1}}+W_{\mathrm{f}, \mathbf{2}}+W_{\mathrm{f}, \mathbf{3}^{\prime}}^{\phi}$. Although the configuration (6) remains an extremum even if we allow ourselves to include mixings, as shown in the previous section, we focus on the case without such mixings for simplicity. Second, many other ways of contraction of the flavon with the same power are also allowed, but it may be natural that some of these possible terms have relatively large numerical coefficients. The superpotential presented in (35)-(39) should be regarded as one of the examples. Our purpose here is to give an existence proof of consistent parameter regions where flavons are correctly stabilized.

In order to stabilize flavon fields with nonzero VEVs, we add negative SUSY breaking masses for flavon fields

$$
\begin{aligned}
V_{\mathrm{SB}}= & -m_{\ell}^{2} \sum_{i=1}^{3}\left|\phi_{\ell, i}\right|^{2}-m_{\mathbf{1}}^{2}\left|\phi_{\mathbf{1}}\right|^{2}-m_{\mathbf{2}}^{2} \sum_{i=1}^{2}\left|\phi_{\mathbf{2}, i}\right|^{2} \\
& -m_{\mathbf{3}^{\prime}}^{2} \sum_{i=1}^{3}\left|\phi_{\mathbf{3}^{\prime}, i}\right|^{2}-m_{\xi}^{2}\left|\xi_{\mathbf{1}}\right|^{2} \\
& -m_{\psi}^{2} \sum_{i=1}^{3}\left|\psi_{\mathbf{3}^{\prime}, i}\right|^{2}-m_{\xi^{\prime}}^{2}\left|\xi_{\mathbf{1}}\right|^{2}
\end{aligned}
$$

Then, the full flavon scalar potential is given by

$$
\begin{aligned}
V= & \sum_{i=1}^{3}\left|\frac{\partial W_{\mathrm{f}, \ell}}{\partial \phi_{\ell, i}}\right|^{2}+\left|\frac{\partial W_{\mathrm{f}, \mathbf{1}}}{\partial \phi_{\mathbf{1}}}\right|^{2}+\sum_{i=1}^{2}\left|\frac{\partial W_{\mathrm{f}, \mathbf{2}}}{\partial \phi_{\mathbf{2}, i}}\right|^{2} \\
& +\sum_{i=1}^{3}\left|\frac{\partial W_{\mathrm{f}, \mathbf{3}^{\prime}}^{\phi}}{\partial \phi_{\mathbf{3}^{\prime}, i}}\right|^{2}+\left|\frac{\partial W_{\mathrm{f}, \xi}}{\partial \xi_{\mathbf{1}}}\right|^{2}+\sum_{i=1}^{3}\left|\frac{\partial W_{\mathrm{f}, \mathbf{3}^{\prime}}^{\psi}}{\partial \psi_{\mathbf{3}^{\prime}, i}}\right|^{2} \\
& +\left|\frac{\partial W_{\mathrm{f}, \xi^{\prime}}}{\partial \xi_{\mathbf{1}}^{\prime}}\right|^{2}+V_{\mathrm{SB}}+V_{A},
\end{aligned}
$$

where $V_{A}$ is the so-called $A$-term potential induced by the supergravity effects

$$
\begin{aligned}
V_{A}= & \frac{3 A}{\Lambda^{3}}\left[h_{1}\left(\phi_{\ell}^{3}\right)_{\mathbf{1}^{\prime}}^{2}+h_{2}\left(\phi_{\ell}^{2}\right)_{\mathbf{1}}\left(\left(\phi_{\ell}^{2}\right)_{\mathbf{3}^{\prime}}^{2}\right)_{\mathbf{1}}+g_{0} \boldsymbol{\phi}_{\mathbf{1}}^{6}+g_{1}\left(\boldsymbol{\phi}_{\mathbf{2}}^{2}\right)_{\mathbf{1}}^{3}\right. \\
& +g_{2}\left(\phi_{\mathbf{2}}^{3}\right)_{\mathbf{1}}^{2}+g_{3}\left(\phi_{\mathbf{3}^{\prime}}^{2}\right)_{\mathbf{1}}^{3}+g_{4}\left(\left[\boldsymbol{\phi}_{\mathbf{3}^{\prime}}^{5}\right] \boldsymbol{\phi}_{\mathbf{3}^{\prime}}\right)_{\mathbf{1}}+g_{\xi} \xi_{\mathbf{1}}^{6} \\
& \left.+g_{5}\left(\psi_{\mathbf{3}^{\prime}}^{2}\right)_{\mathbf{1}}^{3}+g_{6}\left(\left[\psi_{\mathbf{3}^{\prime}}^{5}\right] \psi_{\mathbf{3}^{\prime}}\right)_{\mathbf{1}}+g_{\xi^{\prime}} \boldsymbol{\xi}_{\mathbf{1}}^{\prime 6}\right]+ \text { H.c. }
\end{aligned}
$$

where $|A| \sim m_{3 / 2}$ with $m_{3 / 2}$ being the gravitino mass. Now we check that this model possesses a desired set of vacuum expectation values given in (3) and (6) depending on the choice of parameters $h_{i}(i=1,2)$ and $g_{i}(i=0, \ldots, 6)$. For simplicity, we take all coupling constants $h_{i}$ and $g_{i}$ real. We discuss below the stabilization of $\phi_{1}, \phi_{\ell}, \phi_{2}, \phi_{3^{\prime}}$, and $\psi_{3^{\prime}}$ in order.

\section{A. Potential of $\phi_{1}$}

First, we consider the stabilization of $\phi_{1}$. For the moment, we neglect the contribution from $V_{A}$, which is justified if $m_{3 / 2} \ll m_{1}$. We find a minimum of the potential at

$$
v_{\mathbf{1}}=\left(\frac{1}{180}\right)^{1 / 8}\left(\frac{m_{1} \Lambda^{3}}{\left|g_{0}\right|}\right)^{1 / 4}
$$

Expanding $\phi_{1}$ around the VEV as

$$
\phi_{1}=v_{1}+\frac{1}{\sqrt{2}}\left(\phi_{1}^{R}+i \phi_{1}^{I}\right),
$$

we find that $\phi_{1}^{R}$ has a mass of $2 \sqrt{2} m_{1}$. On the other hand, $\phi_{1}^{I}$ is massless as far as the $A$-term contribution is neglected. This is expected since the scalar potential 
has a global U(1) symmetry and there should be a NambuGoldstone mode when we neglect the $A$ term. After including the $A$ term, $\phi_{1}^{I}$ obtains positive mass squared of the order of $\left|A m_{1}\right|$ for $A>0(A<0)$ when $g_{0}<0$ $\left(g_{0}>0\right)$. Notice that the stabilizations of $\xi_{1}$ and $\xi_{1}^{\prime}$ are completely parallel to $\phi_{1}$.

\section{B. Potential of $\phi_{\ell}$}

Here, we consider the stabilization of $\phi_{\ell}$. As shown in Sec. IV, we can always find an extremum of the form of (3) with

$$
v_{\ell}=\left(\frac{1}{2880}\right)^{1 / 8}\left(\frac{m_{\ell} \Lambda^{3}}{\left|h_{1}\right|}\right)^{1 / 4} .
$$

Actually, the phase of $v_{\ell}$ is fixed after the $A$ term is taken into account, as we see below. If all the mass eigenvalues of the flavon fluctuations around this extremum are positive, this extremum is, in fact, a (local) minimum. In order to see this, we expand the flavon fields as

$$
\begin{aligned}
& \phi_{\ell, 1}=\frac{1}{\sqrt{2}}\left(\phi_{\ell, 1}^{R}+i \phi_{\ell, 1}^{I}\right), \\
& \phi_{\ell, 2}=v_{\ell}+\frac{1}{\sqrt{2}}\left(\phi_{\ell, 2}^{R}+i \phi_{\ell, 2}^{I}\right), \\
& \phi_{\ell, 3}=\frac{1}{\sqrt{2}}\left(\phi_{\ell, 3}^{R}+i \phi_{\ell, 3}^{I}\right) .
\end{aligned}
$$

Then we find out that the mass matrix becomes block diagonal, where the mass for the real and imaginary parts of $\phi_{\ell}$ are separated from each other. As for the real part of the flavon fields, it can be seen that $\phi_{\ell, 2}^{R}$ is a mass eigenstate with mass $2 \sqrt{2} m_{\ell}$, while the other two modes possess a mass matrix of the form of (B4) with

$$
\begin{gathered}
B=-\frac{4\left(9 h_{1}^{2}-3 h_{1} h_{2}-h_{2}^{2}\right)}{45 h_{1}^{2}} m_{\ell}^{2}, \\
C=-\frac{4\left(3 h_{1}+2 h_{2}\right)}{15 h_{1}} m_{\ell}^{2} .
\end{gathered}
$$

Then, the required condition for both of the two mass eigenvalues to be positive is

$h_{2}\left(9 h_{1}+h_{2}\right)>0$ and $\left(6 h_{1}-h_{2}\right)\left(3 h_{1}+h_{2}\right)<0$.

Next, let us turn to the imaginary part of the flavon fields $\phi_{\ell, i}^{I}(i=1,3)$. They possess the mass matrix that is the same as that of the real part but the sign of $C$ is flipped. As a result, the same condition (51) ensures the stability of the corresponding mass eigenstates. On the other hand, $\phi_{\ell, 2}^{I}$ becomes massless at this approximation but $V_{A}$ gives the leading contribution to its mass. If we write the VEV of the flavon as $\left\langle\phi_{\ell, 2}\right\rangle=v_{\ell} e^{i \varphi}$, the potential is minimized at $\varphi=$ $2 n \pi / 3(n=0,1,2)$ for $A>0(A<0)$ when $h_{1}<0$ $\left(h_{1}>0\right)$. In Eq. (47) we just chose $n=0$ solution. A similar argument should be understood in the following analysis of $\phi_{2}, \phi_{3^{\prime}}$, and $\psi_{3^{\prime}}$. In the upper left part of Fig. 2, we show the allowed region in the $h_{1}$ vs $h_{2}$ plane as a blue region.

\section{Potential of $\phi_{2}$}

Now, we consider the $\phi_{2}$ sector of the potential. There exists an extremum of the form of (6), irrespective of the choice of parameters, with

$$
v_{2}=\left(\frac{1}{720}\right)^{1 / 8}\left(\frac{m_{2} \Lambda^{3}}{\left|2 g_{1}+g_{2}\right|}\right)^{1 / 4} .
$$

Expanding the flavon fields as

$$
\begin{aligned}
& \phi_{2,1}=v_{2}+\frac{1}{\sqrt{2}}\left(\phi_{2,1}^{R}+i \phi_{2,1}^{I}\right), \\
& \phi_{2,2}=v_{2}+\frac{1}{\sqrt{2}}\left(\phi_{2,2}^{R}+i \phi_{2,2}^{I}\right),
\end{aligned}
$$

we find the mass matrix being block diagonal when we neglect the contribution from $V_{A}$. The mass matrix for the real part is given by (B4) with

$$
\begin{gathered}
B=\frac{128 g_{1}^{2}+140 g_{1} g_{2}+47 g_{2}^{2}}{10\left(2 g_{1}+g_{2}\right)^{2}} m_{2}^{2}, \\
C=\frac{3\left(64 g_{1}^{2}+60 g_{1} g_{2}+11 g_{2}^{2}\right)}{10\left(2 g_{1}+g_{2}\right)^{2}} m_{2}^{2},
\end{gathered}
$$

and the condition for both mass eigenvalues to be positive is given by

$$
\left(4 g_{1}-g_{2}\right)\left(8 g_{1}+7 g_{2}\right)<0 .
$$

On the other hand, the mass matrix for the imaginary part is also given by (B4) but with

$$
B=-\frac{9 g_{2}\left(4 g_{1}+g_{2}\right)}{10\left(2 g_{1}+g_{2}\right)^{2}} m_{2}^{2}=-C .
$$

As mentioned in Appendix B, this mass matrix possesses a massless mode and the condition for another mass eigenstate to have positive mass squared is

$$
g_{2}\left(4 g_{1}+g_{2}\right)<0 \text {. }
$$

As for the massless mode, the potential $V_{A}$ neglected so far gives it a nonzero mass squared, whose sign becomes positive if $A>0 \quad(A<0)$ when $2 g_{1}+g_{2}<0$ $\left(2 g_{1}+g_{2}>0\right)$. In the upper right part of Fig. 2, we show the allowed region in the $g_{1}$ vs $g_{2}$ plane. 

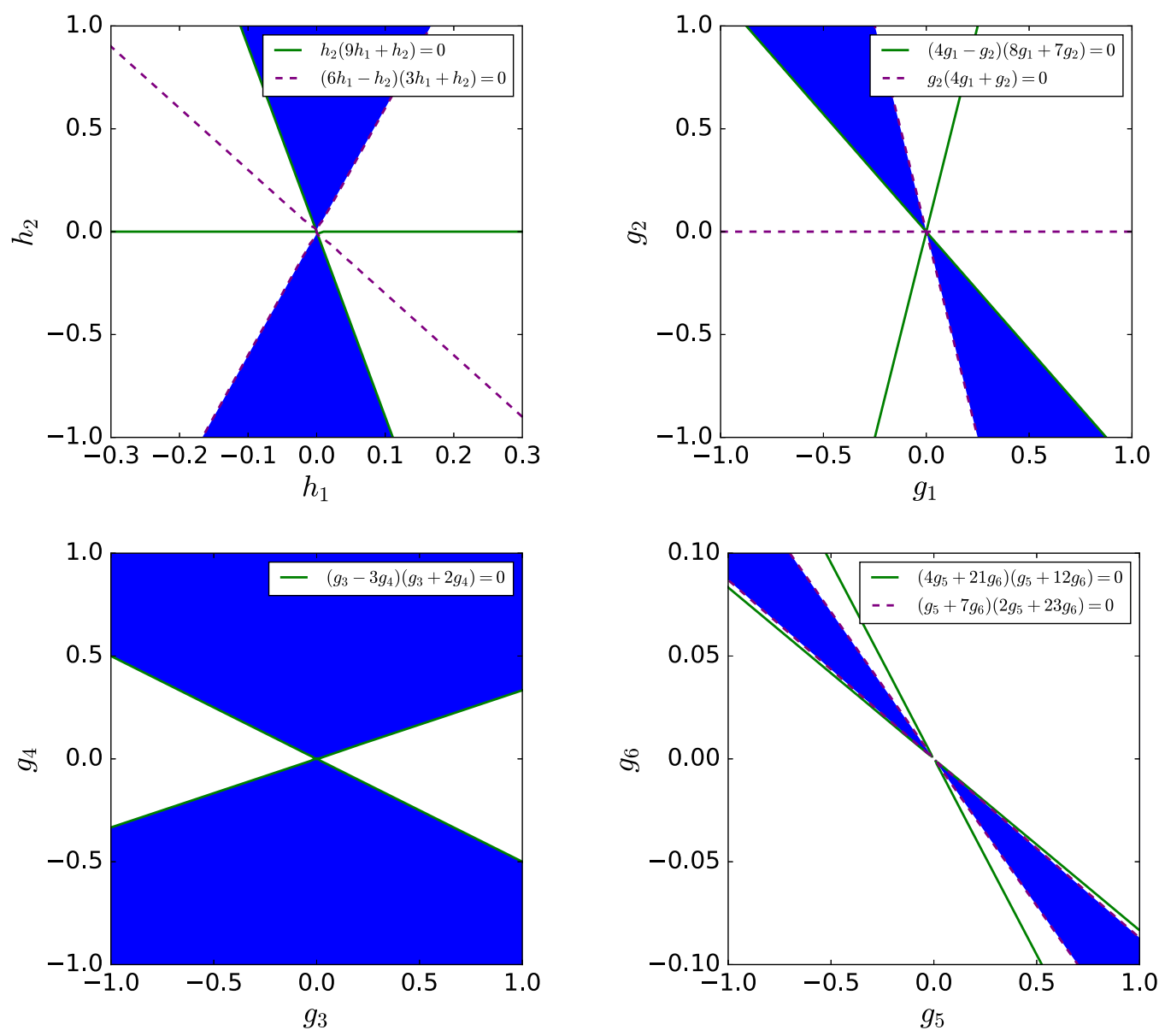

FIG. 2. The stabilization conditions of the flavon fields imposed on the parameter space. In each figure, the blue region is allowed by the conditions. Figures correspond to the parameter space of the $\phi_{\ell}$ sector (upper left), the $\phi_{2}$ sector (upper right), the $\phi_{3^{\prime}}$ sector (lower left), and the $\psi_{\mathbf{3}^{\prime}}$ sector (lower right). For the relevant conditions, see (51), (56), (65), (72), and (76).

\section{Potential of $\phi_{3^{\prime}}$}

Let us move on to the $\phi_{3^{\prime}}$ sector of the potential. We can find an extremum at the position of (6), with $v_{3^{\prime}}$ given by

$$
v_{3^{\prime}}=\left(\frac{1}{14580}\right)^{1 / 8}\left(\frac{m_{3^{\prime}} \Lambda^{3}}{\left|g_{3}\right|}\right)^{1 / 4} .
$$

Expanding the flavon fields around the extremum as

$$
\begin{aligned}
& \phi_{3^{\prime}, 1}=v_{3^{\prime}}+\frac{1}{\sqrt{2}}\left(\phi_{3^{\prime}, 1}^{R}+i \phi_{3^{\prime}, 1}^{I}\right), \\
& \phi_{3^{\prime}, 2}=v_{3^{\prime}}+\frac{1}{\sqrt{2}}\left(\phi_{3^{\prime}, 2}^{R}+i \phi_{3^{\prime}, 2}^{I}\right), \\
& \phi_{3^{\prime}, 3}=v_{3^{\prime}}+\frac{1}{\sqrt{2}}\left(\phi_{3^{\prime}, 3}^{R}+i \phi_{3^{\prime}, 3}^{I}\right),
\end{aligned}
$$

we obtain a block diagonal mass matrix for flavon fields when we neglect the small contribution from $V_{A}$. The mass matrix for the real part of $\phi_{3^{\prime}}$ takes the form of (B6) with

$$
D=\frac{8\left(5 g_{3}^{2}+18 g_{3} g_{4}+36 g_{4}^{2}\right)}{15 g_{3}^{2}} m_{3^{\prime}}^{2},
$$

$$
\begin{gathered}
E=\frac{8\left(g_{3}-3 g_{4}\right)\left(5 g_{3}+6 g_{4}\right)}{15 g_{3}^{2}} m_{\mathbf{3}^{\prime}}^{2}, \\
F=\frac{4\left(7 g_{3}^{2}+72 g_{4}^{2}\right)}{15 g_{3}^{2}} m_{\mathbf{3}^{\prime}}^{2}, \\
G=\frac{4\left(13 g_{3}^{2}+18 g_{3} g_{4}-36 g_{4}^{2}\right)}{15 g_{3}^{2}} m_{\mathbf{3}^{\prime}}^{2} .
\end{gathered}
$$

We find that, if the coupling parameters satisfy the conditions

$$
\left(g_{3}-3 g_{4}\right)\left(g_{3}+2 g_{4}\right)<0,
$$

all the eigenvalues become positive. For the imaginary part of $\phi_{3^{\prime}}$, the same conditions ensure the stability of all the eigenstates but one, which becomes massless. Again, the dominant contribution to its mass comes from $V_{A}$, whose sign becomes positive if $A>0(A<0)$ when $g_{3}<0$ $\left(g_{3}>0\right)$. In the lower left part of Fig. 2, we show the allowed region in the $g_{3}$ vs $g_{4}$ plane. 


\section{E. Potential of $\psi_{3^{\prime}}$}

The form of the potential of $\psi_{3^{\prime}}$ is exactly the same as that of $\phi_{3^{\prime}}$. However, as we see below, it is possible for the potential to possess a required form of the vacuum depending on the choice of the coupling parameters. As in the other sectors, we can find an extremum of the form of (6) irrespective of the coupling parameters, with $v_{\psi}$ expressed as

$$
v_{\psi}=\left(\frac{1}{2880}\right)^{1 / 8}\left(\frac{m_{\psi} \Lambda^{3}}{\left|g_{5}+9 g_{6}\right|}\right)^{1 / 4}
$$

We expand the flavon fields around the extremum as

$$
\begin{aligned}
& \psi_{3^{\prime}, 1}=\frac{1}{\sqrt{2}}\left(\psi_{3^{\prime}, 1}^{R}+i \psi_{3^{\prime}, 1}^{I}\right), \\
& \psi_{3^{\prime}, 2}=v_{\psi}+\frac{1}{\sqrt{2}}\left(\psi_{3^{\prime}, 2}^{R}+i \psi_{3^{\prime}, 2}^{I}\right), \\
& \psi_{3^{\prime}, 3}=-v_{\psi}+\frac{1}{\sqrt{2}}\left(\psi_{3^{\prime}, 3}^{R}+i \psi_{3^{\prime}, 3}^{I}\right),
\end{aligned}
$$

and calculate the mass matrix of the real and imaginary parts of $\psi_{3^{\prime}}$. For the real part, we obtain a matrix of the form of (B6) with

$$
\begin{gathered}
D=\frac{-\left(8 g_{5}^{2}+138 g_{5} g_{6}+423 g_{6}^{2}\right)}{5\left(g_{5}+9 g_{6}\right)^{2}} m_{\psi}^{2}, \\
E=\frac{-9 g_{6}\left(2 g_{5}+15 g_{6}\right)}{5\left(g_{5}+9 g_{6}\right)^{2}} m_{\psi}^{2}, \\
F=\frac{16 g_{5}^{2}+282 g_{5} g_{6}+1341 g_{6}^{2}}{5\left(g_{5}+9 g_{6}\right)^{2}} m_{\psi}^{2}, \\
G=\frac{-3\left(8 g_{5}^{2}+146 g_{5} g_{6}+633 g_{6}^{2}\right)}{5\left(g_{5}+9 g_{6}\right)^{2}} m_{\psi}^{2},
\end{gathered}
$$

all of whose eigenvalues become positive if

$$
\left(4 g_{5}+21 g_{6}\right)\left(g_{5}+12 g_{6}\right)<0 .
$$

Similarly, the mass matrix for the imaginary part is given by

$$
\begin{gathered}
D=\frac{-9 g_{6}\left(2 g_{5}-g_{6}\right)}{5\left(g_{5}+9 g_{6}\right)^{2}} m_{\psi}^{2}, \\
E=\frac{27 g_{6}\left(2 g_{5}+19 g_{6}\right)}{5\left(g_{5}+9 g_{6}\right)^{2}} m_{\psi}^{2}, \\
F=G=\frac{9 g_{6}\left(2 g_{5}+29 g_{6}\right)}{5\left(g_{5}+9 g_{6}\right)^{2}} m_{\psi}^{2},
\end{gathered}
$$

and the conditions for all the eigenvalues to be positive are

$$
\left(g_{5}+7 g_{6}\right)\left(2 g_{5}+23 g_{6}\right)<0,
$$

together with the condition for the stabilization of one of the eigenstates by $V_{A}: A>0(A<0)$ when $g_{5}+9 g_{6}>0$ $\left(g_{5}+9 g_{6}<0\right)$. In the lower right part of Fig. 2, we show the allowed region in the $g_{5}$ vs $g_{6}$ plane.

\section{F. Fermion sector}

Finally, we briefly comment on the masses of fermionic partners of the flavon fields (flavinos). By diagonalizing their mass matrices, we can easily check that all the mass eigenstates generally possess masses of the order of the SUSY breaking mass scale. Below we list all the mass eigenstates and the corresponding mass eigenvalues. For the $S_{4}$ singlet flavon sector, we have

$$
\begin{array}{ll}
\tilde{\phi}_{1} ; & m=\operatorname{sgn}\left(g_{0}\right) \sqrt{5} m_{1}, \\
\tilde{\xi}_{1} ; & m=\operatorname{sgn}\left(g_{\xi}\right) \sqrt{5} m_{\xi}, \\
\tilde{\xi}_{1}^{\prime} ; & m=\operatorname{sgn}\left(g_{\xi^{\prime}}\right) \sqrt{5} m_{\xi^{\prime}},
\end{array}
$$

where the tilde denotes the fermionic superpartner of any scalar field. For the $\phi_{\ell}$ sector, we have

$$
\begin{gathered}
\tilde{\phi}_{\ell, 2} ; \quad m=\operatorname{sgn}\left(h_{1}\right) \sqrt{5} m_{\ell}, \\
\frac{1}{\sqrt{2}}\left(\tilde{\phi}_{\ell, 1} \pm \tilde{\phi}_{\ell, 3}\right) ; \quad m= \pm \frac{\left(3 h_{1}+2 h_{2}\right)}{3 \sqrt{5}\left|h_{1}\right|} m_{\ell} .
\end{gathered}
$$

For the $\phi_{2}$ sector, we have

$$
\begin{array}{r}
\frac{1}{\sqrt{2}}\left(\tilde{\phi}_{2,1}+\tilde{\phi}_{2,2}\right) ; \quad m=\operatorname{sgn}\left(2 g_{1}+g_{2}\right) \sqrt{5} m_{\mathbf{2}}, \\
\frac{1}{\sqrt{2}}\left(\tilde{\phi}_{2,1}-\tilde{\phi}_{2,2}\right) ; \quad m=\frac{2\left(g_{1}-g_{2}\right)}{\sqrt{5}\left|2 g_{1}+g_{2}\right|} m_{\mathbf{2}} .
\end{array}
$$

Finally, for the $\phi_{3^{\prime}}$ sector, we obtain

$$
\begin{gathered}
\frac{1}{\sqrt{3}}\left(\tilde{\phi}_{3^{\prime}, 1}+\tilde{\phi}_{3^{\prime}, 2}+\tilde{\phi}_{\mathbf{3}^{\prime}, 3}\right) ; \quad m=\sqrt{5} m_{\mathbf{3}^{\prime}}, \\
\frac{1}{\sqrt{2}}\left(\tilde{\phi}_{\mathbf{3}^{\prime}, 2}-\tilde{\phi}_{3^{\prime}, 3}\right) ; \quad m=-\frac{\left(g_{3}+12 g_{4}\right)}{\sqrt{5}\left|g_{3}\right|} m_{\mathbf{3}^{\prime}}, \\
\frac{1}{\sqrt{6}}\left(-2 \tilde{\phi}_{\mathbf{3}^{\prime}, 1}+\tilde{\phi}_{\mathbf{3}^{\prime}, 2}+\tilde{\phi}_{\mathbf{3}^{\prime}, 3}\right) ; \quad m=\frac{\left(g_{3}+12 g_{4}\right)}{\sqrt{5}\left|g_{3}\right|} m_{\mathbf{3}^{\prime}},
\end{gathered}
$$

and for $\psi_{3^{\prime}}$ sectors, 


$$
\begin{aligned}
& \frac{1}{\sqrt{3}}\left(\tilde{\psi}_{3^{\prime}, 1}+\tilde{\psi}_{3^{\prime}, 2}+\tilde{\psi}_{3^{\prime}, 3}\right) ; \quad m=\frac{\left(g_{5}+24 g_{6}\right)}{\sqrt{5}\left|g_{5}+9 g_{6}\right|} m_{\psi}, \\
& \frac{1}{\sqrt{2}}\left(\tilde{\psi}_{3^{\prime}, 2}-\tilde{\psi}_{3^{\prime}, 3}\right) ; \quad m=-\operatorname{sgn}\left(g_{5}+24 g_{6}\right) \sqrt{5} m_{\psi}, \\
& \frac{1}{\sqrt{6}}\left(-2 \tilde{\psi}_{3^{\prime}, 1}+\tilde{\psi}_{3^{\prime}, 2}+\tilde{\psi}_{3^{\prime}, 3}\right) ; \quad m=\frac{\left(g_{5}-3 g_{6}\right)}{\sqrt{5}\left|g_{5}+9 g_{6}\right|} m_{\psi} .
\end{aligned}
$$

All flavinos have masses of the order of the SUSY breaking mass and hence it may be possible that one of them is the lightest SUSY particle and a candidate of dark matter. There are several ways to produce flavinos in the early Universe, for example, by thermal scattering of minimal SUSY standard model (MSSM) particles or the decay of MSSM particles, gravitino, flavons, and so on.

\section{CONCLUSIONS AND DISCUSSION}

An $S_{4}$ flavor model can lead to the so-called $\mathrm{TM}_{1}$ pattern of neutrino mixings consistent with current experimental data if all the flavons are stabilized appropriately. We have explicitly constructed a model in which all flavons have VEVs with the desired alignment structure in a simple way. The flavon stabilization is achieved by the balance between the tachyonic SUSY breaking mass and the higher-dimensional terms in the potential. In our model, we do not need any additional field (such as the driving fields) in order to stabilize flavons. In this sense, our model is very simple. In addition, although we study an $S_{4}$ model in this paper, this mechanism is universal and can be applied to many flavor models based on discrete flavor symmetry.

Having seen that the desired flavon VEV alignments (3) and (6) can be obtained in our setup, we shortly discuss the implications for the cosmological domain wall problem. During inflation, the flavons are stabilized due to the negative Hubble-induced mass terms [45] instead of the tachyonic SUSY breaking mass terms, but the VEV alignment structure is exactly the same as that by the latter. Once the flavons settle down in the desired minimum of the potential during inflation, they remain trapped in the varying minimum after inflation when the Hubble parameter $H$ gradually decreases. The flavons finally get into the present vacuum when $H$ becomes comparable to the SUSY breaking mass. One can show that flavons do not overshoot the origin of the field space during this whole cosmological dynamics as shown in Ref. [22]. Thus in this scenario, the discrete flavor symmetry is already spontaneously broken during inflation and never restored thereafter, which implies that there is no cosmological domain wall problem.

So far we have neglected thermal effects on the flavon potential. The existence of high-temperature plasma in the early Universe can affect the flavon potential that might lead to the symmetry restoration. For concreteness, let us consider a standard cosmological scenario that the Universe enters in the matter domination era due to the coherent inflaton oscillation after the end of inflation and finally the inflaton decays and the radiation-dominated universe begins at $T=T_{\mathrm{R}}$, where $T$ is the radiation temperature and $T_{\mathrm{R}}$ is the reheating temperature. In our model, flavons do not have renormalizable interactions with MSSM fields. Still, nonrenormalizable interactions may give rise to sizable thermal effects. The dominant thermal effect on the flavon potential comes from the tau Yukawa coupling in (2). It arises at the two-loop order,

$$
V_{T} \sim \frac{y_{\tau}^{2} T^{4}}{\Lambda^{2}}\left|\phi_{\ell}\right|^{2}
$$

In order for this thermal potential not to affect the flavon dynamics significantly, we demand that the thermal potential is always subdominant compared with either the Hubble mass term $H^{2}\left|\phi_{\ell}\right|^{2}$ or the SUSY breaking mass term $m_{\ell}^{2}\left|\phi_{\ell}\right|^{2}$. Assuming $T_{\mathrm{R}} \lesssim \sqrt{m_{\ell} M_{\mathrm{P}}}$, where $M_{\mathrm{P}}$ is the reduced Planck scale, it is sufficient to demand that

$$
\begin{aligned}
\left.\frac{y_{\tau}^{2} T^{4}}{\Lambda^{2}}\right|_{H=m_{\ell}} \lesssim & m_{\ell}^{2} \leftrightarrow y_{\tau}^{2}\left(\frac{T_{\mathrm{R}}}{10^{6} \mathrm{GeV}}\right)^{2}\left(\frac{10^{6} \mathrm{GeV}}{m_{\ell}}\right) \\
& \times\left(\frac{10^{12} \mathrm{GeV}}{\Lambda}\right)^{2} \lesssim 1,
\end{aligned}
$$

where we have used $T^{4} \sim T_{\mathrm{R}}^{2} H M_{\mathrm{P}}$ before the completion of the reheating. If this condition is satisfied, thermal effects on the flavon dynamics are safely neglected. ${ }^{6}$ Thermal effects on the other flavons, $\phi_{1}, \phi_{2}, \phi_{3^{\prime}}$, and $\psi_{3^{\prime}}$, are suppressed by an additional power of $(T / \Lambda)^{2}$ and hence are negligible. Therefore, as far as the reheating temperature is not too high, our model is cosmologically viable.

\section{ACKNOWLEDGMENTS}

This work was supported by the JSPS KAKENHI Grant No. 17J00813 (S. C.), Grant-in-Aid for Scientific Research C Grant No. 18K03609 (K. N.), and Innovative Areas Grants No. 15H05888 (K. N.) and No. 17H06359 (K. N.).

\section{APPENDIX A: NOTES ON $S_{4}$ REPRESENTATIONS}

In this Appendix, we summarize the representation of the $S_{4}$ group and product rules. Our convention is the same as, e.g., the Appendix of Ref. [37].

\footnotetext{
${ }^{6}$ Although it does not lead to the symmetry restoration, a small amount of flavon oscillation around its potential minimum may be induced [46-49].
} 
All the elements of the $S_{4}$ group can be written as a product of three elements often called $S, U$, and $T$, which generates $Z_{2}$, another $Z_{2}$, and $Z_{3}$ subgroup of $S_{4}$, respectively. $S_{4}$ has five different representations: $\mathbf{1}, \mathbf{1}^{\prime}, \mathbf{2}, \mathbf{3}$, and $\mathbf{3}^{\prime}$, where the number denotes the dimension of each representation. In doublet representation 2 , representation matrices for generators are given as

$$
S=\left(\begin{array}{ll}
1 & 0 \\
0 & 1
\end{array}\right), \quad U=\left(\begin{array}{cc}
0 & 1 \\
1 & 0
\end{array}\right), \quad T=\left(\begin{array}{cc}
\omega & 0 \\
0 & \omega^{2}
\end{array}\right),
$$

where we define $\omega \equiv e^{2 \pi i / 3}$. In triplet representations $\mathbf{3}$ and $\mathbf{3}^{\prime}$, corresponding matrices are

$$
\begin{aligned}
S & =\frac{1}{3}\left(\begin{array}{ccc}
-1 & 2 & 2 \\
2 & -1 & 2 \\
2 & 2 & -1
\end{array}\right), \quad U=\mp\left(\begin{array}{lll}
1 & 0 & 0 \\
0 & 0 & 1 \\
0 & 1 & 0
\end{array}\right), \\
T & =\left(\begin{array}{ccc}
1 & 0 & 0 \\
0 & \omega^{2} & 0 \\
0 & 0 & \omega
\end{array}\right),
\end{aligned}
$$

where $-(+)$ for the $\mathbf{3}\left(\mathbf{3}^{\prime}\right)$ representation.

In order to show the product rules in this basis, we define a nontrivial singlet $p^{\prime}$ in $\mathbf{1}^{\prime}$, a doublet $a=\left(a_{1}, a_{2}\right)$, and two triplets $b=\left(b_{1}, b_{2}, b_{3}\right)$ in $\mathbf{3}$ and $b^{\prime}=\left(b_{1}^{\prime}, b_{2}^{\prime}, b_{3}^{\prime}\right)$ in $\mathbf{3}^{\prime}$. We also use a tilde in order to use another multiplet in the same representation. First, products with $\mathbf{1}^{\prime}$ are decomposed as $\mathbf{1}^{\prime} \times \mathbf{1}^{\prime}=\mathbf{1}, \mathbf{2} \times \mathbf{1}^{\prime}=2,3 \times \mathbf{1}^{\prime}=3^{\prime}$, and $3^{\prime} \times \mathbf{1}^{\prime}=3$ : in components,

$$
\begin{aligned}
& \left(p^{\prime} \tilde{p}^{\prime}\right)_{\mathbf{1}}=p^{\prime} \tilde{p}^{\prime}, \quad\left(p^{\prime} a\right)_{\mathbf{2}}=\left(\begin{array}{c}
p^{\prime} a_{1} \\
-p^{\prime} a_{2}
\end{array}\right), \\
& \left(p^{\prime} b\right)_{\mathbf{3}^{\prime}}=\left(\begin{array}{l}
p^{\prime} b_{1} \\
p^{\prime} b_{2} \\
p^{\prime} b_{3}
\end{array}\right), \quad\left(p^{\prime} b^{\prime}\right)_{\mathbf{3}}=\left(\begin{array}{l}
p^{\prime} b_{1}^{\prime} \\
p^{\prime} b_{2}^{\prime} \\
p^{\prime} b_{3}^{\prime}
\end{array}\right),
\end{aligned}
$$

where the parenthesis denotes the contraction of several representations that as a whole transforms as a representation denoted by the subscript. Next, the product of a doublet with another doublet is decomposed as $\mathbf{2} \times \mathbf{2}=$ $\mathbf{1}+\mathbf{1}^{\prime}+\mathbf{2}$ and

$$
\begin{aligned}
& (a \tilde{a})_{\mathbf{1}}=a_{1} \tilde{a}_{2}+a_{2} \tilde{a}_{1}, \quad(a \tilde{a})_{\mathbf{1}^{\prime}}=a_{1} \tilde{a}_{2}-a_{2} \tilde{a}_{1}, \\
& (a \tilde{a})_{\mathbf{2}}=\left(\begin{array}{c}
a_{2} \tilde{a}_{2} \\
a_{1} \tilde{a}_{1}
\end{array}\right),
\end{aligned}
$$

while that with a triplet is $\mathbf{2} \times \mathbf{3}^{(')}=\mathbf{3}+\mathbf{3}^{\prime}$ and

$$
\begin{aligned}
\left(a b^{(\prime)}\right)_{\mathbf{3}} & =\left(\begin{array}{l}
a_{1} b_{2}^{(\prime)} \pm a_{2} b_{3}^{(\prime)} \\
a_{1} b_{3}^{(\prime)} \pm a_{2} b_{1}^{(\prime)} \\
a_{1} b_{1}^{(\prime)} \pm a_{2} b_{2}^{(\prime)}
\end{array}\right), \\
\left(a b^{(\prime)}\right)_{\mathbf{3}^{\prime}} & =\left(\begin{array}{l}
a_{1} b_{2}^{(\prime)} \mp a_{2} b_{3}^{(\prime)} \\
a_{1} b_{3}^{(\prime)} \mp a_{2} b_{1}^{(\prime)} \\
a_{1} b_{1}^{(\prime)} \mp a_{2} b_{2}^{(\prime)}
\end{array}\right),
\end{aligned}
$$

where the upper (lower) sign is for $\mathbf{3}\left(\mathbf{3}^{\prime}\right)$. The product of two triplets is decomposed as $\mathbf{3} \times \mathbf{3}=\mathbf{1}+\mathbf{2}+\mathbf{3}+\mathbf{3}^{\prime}$ and

$$
\begin{aligned}
& (b \tilde{b})_{\mathbf{1}}=b_{1} \tilde{b}_{1}+b_{2} \tilde{b}_{3}+b_{3} \tilde{b}_{2}, \\
& (b \tilde{b})_{\mathbf{2}}=\left(\begin{array}{l}
b_{1} \tilde{b}_{3}+b_{2} \tilde{b}_{2}+b_{3} \tilde{b}_{1} \\
b_{1} \tilde{b}_{2}+b_{2} \tilde{b}_{1}+b_{3} \tilde{b}_{3}
\end{array}\right), \\
& (b \tilde{b})_{\mathbf{3}}=\left(\begin{array}{l}
b_{2} \tilde{b}_{3}-b_{3} \tilde{b}_{2} \\
b_{1} \tilde{b}_{2}-b_{2} \tilde{b}_{1} \\
b_{3} \tilde{b}_{1}-b_{1} \tilde{b}_{3}
\end{array}\right), \\
& (b \tilde{b})_{\mathbf{3}^{\prime}}=\left(\begin{array}{c}
2 b_{1} \tilde{b}_{1}-b_{2} \tilde{b}_{3}-b_{3} \tilde{b}_{2} \\
-b_{1} \tilde{b}_{2}-b_{2} \tilde{b}_{1}+2 b_{3} \tilde{b}_{3} \\
-b_{1} \tilde{b}_{3}+2 b_{2} \tilde{b}_{2}-b_{3} \tilde{b}_{1}
\end{array}\right) .
\end{aligned}
$$

The product $\mathbf{3}^{\prime} \times \mathbf{3}^{\prime}$ is decomposed in the same way and the component product rules can be obtained by substituting $(b, \tilde{b}) \rightarrow\left(b^{\prime}, \tilde{b}^{\prime}\right)$ in (A6). Finally, the remaining nontrivial product is $\mathbf{3} \times \mathbf{3}^{\prime}=\mathbf{1}^{\prime}+\mathbf{2}+\mathbf{3}+\mathbf{3}^{\prime}$ : in components,

$$
\begin{aligned}
\left(b b^{\prime}\right)_{\mathbf{1}^{\prime}}= & b_{1} b_{1}^{\prime}+b_{2} b_{3}^{\prime}+b_{3} b_{2}^{\prime}, \\
\left(b b^{\prime}\right)_{\mathbf{2}}= & \left(\begin{array}{c}
b_{1} b_{3}^{\prime}+b_{2} b_{2}^{\prime}+b_{3} b_{1}^{\prime} \\
-b_{1} b_{2}^{\prime}-b_{2} b_{1}^{\prime}-b_{3} b_{3}^{\prime}
\end{array}\right), \\
\left(b b^{\prime}\right)_{\mathbf{3}}= & \left(\begin{array}{c}
2 b_{1} b_{1}^{\prime}-b_{2} b_{3}^{\prime}-b_{3} b_{2}^{\prime} \\
-b_{1} b_{2}^{\prime}-b_{2} b_{1}^{\prime}+2 b_{3} b_{3}^{\prime} \\
-b_{1} b_{3}^{\prime}+2 b_{2} b_{2}^{\prime}-b_{3} b_{1}^{\prime}
\end{array}\right), \\
\left(b b^{\prime}\right)_{\mathbf{3}^{\prime}}= & \left(\begin{array}{l}
b_{2} b_{3}^{\prime}-b_{3} b_{2}^{\prime} \\
b_{1} b_{2}^{\prime}-b_{2} b_{1}^{\prime} \\
b_{3} b_{1}^{\prime}-b_{1} b_{3}^{\prime}
\end{array}\right) .
\end{aligned}
$$

\section{APPENDIX B: DIAGONALIZATION OF MASS MATRICES}

\section{Neutrino sector}

The fermion mass matrix $\mathcal{M}$ is in general complex and symmetric. A symmetric complex matrix is diagonalized by a unitary matrix $X$ in the form of $X \mathcal{M} X^{T}$ (Takagi diagonalization [50]). As a concrete example, let us consider $2 \times 2$ complex mass matrix 


$$
\mathcal{M}=\left(\begin{array}{ll}
B & C \\
C & D
\end{array}\right) .
$$

A general unitary matrix may be expressed as

$$
X=\left(\begin{array}{cc}
\cos \theta & e^{i \eta} \sin \theta \\
-e^{-i \eta} \sin \theta & \cos \theta
\end{array}\right)\left(\begin{array}{cc}
e^{i \alpha} & 0 \\
0 & e^{i \beta}
\end{array}\right),
$$

where $\theta, \eta, \alpha$, and $\beta$ are real parameters. We find that $X^{T} \mathcal{M} X$ becomes diagonal if we take

$\tan 2 \theta=\frac{2\left|C^{*} D+C B^{*}\right|}{|D|^{2}-|B|^{2}}, \quad e^{i \eta}=\frac{C^{*} D+C B^{*}}{\left|C^{*} D+C B^{*}\right|}$.

The parameters $\alpha$ and $\beta$ can be fixed if one wants to make the mass eigenvalues real. The neutrino mass matrix in $\mathrm{TM}_{1}$ model (11) can be diagonalized using this expression by identifying $u_{23}=X$.

\section{Flavon sector}

There are only two types of mass matrices that appear in the analysis of the scalar sector of flavon fields. In this Appendix, we summarize all of their eigenvectors and eigenvalues. First, in Secs. V B and VC, we obtain the mass matrices of the form of

$$
\mathcal{M}_{2}^{2}=\left(\begin{array}{ll}
B & C \\
C & B
\end{array}\right),
$$

which is diagonalized by an orthogonal matrix $O$ as

$$
O \mathcal{M}_{2}^{2} O^{T}=\left(\begin{array}{cc}
B-C & 0 \\
0 & B+C
\end{array}\right) ; \quad O=\frac{1}{\sqrt{2}}\left(\begin{array}{cc}
1 & -1 \\
1 & 1
\end{array}\right) .
$$

Note that there exists a massless field if $B= \pm C$. For the analysis of triplet flavon fields in Secs. V D and VE, we obtain the mass matrices of the form of

$$
\mathcal{M}_{3}^{2}=\left(\begin{array}{lll}
D & E & E \\
E & F & G \\
E & G & F
\end{array}\right),
$$

which can be diagonalized as

$$
\begin{aligned}
& V \mathcal{M}_{3}^{2} V^{T} \\
& =\left(\begin{array}{ccc}
F-G & 0 & 0 \\
0 & \frac{1}{2}(D+F+G-\kappa) & 0 \\
0 & 0 & \frac{1}{2}(D+F+G+\kappa)
\end{array}\right),
\end{aligned}
$$

$V=\left(\begin{array}{ccc}0 & -\frac{1}{\sqrt{2}} & \frac{1}{\sqrt{2}} \\ \frac{D-F-G-\kappa}{E N_{-}} & \frac{2}{N_{-}} & \frac{2}{N_{-}} \\ \frac{D-F-G+\kappa}{E N_{+}} & \frac{2}{N_{+}} & \frac{2}{N_{+}}\end{array}\right)$,

for $E \neq 0$, where

$$
\kappa \equiv \sqrt{(D-F-G)^{2}+8 E^{2}},
$$

and $N_{ \pm}$are proper normalization factors with which the squared sum of each line of $V$ becomes 1 . Note that there is a massless mode if $F=G$, as seen in Secs. V D and V E.
[1] G. Altarelli and F. Feruglio, Rev. Mod. Phys. 82, 2701 (2010).

[2] H. Ishimori, T. Kobayashi, H. Ohki, Y. Shimizu, H. Okada, and M. Tanimoto, Prog. Theor. Phys. Suppl. 183, 1 (2010).

[3] F. P. An et al. (Daya Bay Collaboration), Phys. Rev. Lett. 108, 171803 (2012).

[4] J. K. Ahn et al. (RENO Collaboration), Phys. Rev. Lett. 108, 191802 (2012).

[5] S. F. King and C. Luhn, Rep. Prog. Phys. 76, 056201 (2013).

[6] S. F. King, A. Merle, S. Morisi, Y. Shimizu, and M. Tanimoto, New J. Phys. 16, 045018 (2014).

[7] S. F. King, Prog. Part. Nucl. Phys. 94, 217 (2017).

[8] S. F. King, arXiv:1904.06660.

[9] J. Preskill, S. P. Trivedi, F. Wilczek, and M. B. Wise, Nucl. Phys. B363, 207 (1991).

[10] L. E. Ibanez and G. G. Ross, Phys. Lett. B 260, 291 (1991).
[11] L. E. Ibanez and G. G. Ross, Nucl. Phys. B368, 3 (1992).

[12] T. Banks and M. Dine, Phys. Rev. D 45, 1424 (1992).

[13] T. Araki, Prog. Theor. Phys. 117, 1119 (2007).

[14] T. Araki, T. Kobayashi, J. Kubo, S. Ramos-Sanchez, M. Ratz, and P. K. S. Vaudrevange, Nucl. Phys. B805, 124 (2008).

[15] C. Luhn and P. Ramond, J. High Energy Phys. 07 (2008) 085.

[16] M. C. Chen, M. Ratz, and A. Trautner, J. High Energy Phys. 09 (2013) 096.

[17] M. C. Chen, M. Fallbacher, M. Ratz, A. Trautner, and P. K. S. Vaudrevange, Phys. Lett. B 747, 22 (2015).

[18] F. Riva, Phys. Lett. B 690, 443 (2010).

[19] S. Chigusa and K. Nakayama, Phys. Lett. B 788, 249 (2019).

[20] S. Antusch and D. Nolde, J. Cosmol. Astropart. Phys. 10 (2013) 028. 
[21] S. F. King and Y. L. Zhou, J. High Energy Phys. 11 (2018) 173.

[22] S. Chigusa, S. Kasuya, and K. Nakayama, Phys. Lett. B 788, 494 (2019).

[23] Y. Ema, K. Nakayama, and M. Takimoto, J. Cosmol. Astropart. Phys. 02 (2016) 067.

[24] G. Altarelli and F. Feruglio, Nucl. Phys. B741, 215 (2006).

[25] S. Pascoli and Y. L. Zhou, J. High Energy Phys. 06 (2016) 073.

[26] I. de Medeiros Varzielas, T. Neder, and Y. L. Zhou, Phys. Rev. D 97, 115033 (2018).

[27] I. De Medeiros Varzielas, M. Levy, and Y.L. Zhou, arXiv:1903.10506.

[28] R. N. Mohapatra, M. K. Parida, and G. Rajasekaran, Phys. Rev. D 69, 053007 (2004).

[29] N. Haba, A. Watanabe, and K. Yoshioka, Phys. Rev. Lett. 97, 041601 (2006).

[30] X. G. He and A. Zee, Phys. Lett. B 645, 427 (2007).

[31] W. Grimus and L. Lavoura, J. High Energy Phys. 09 (2008) 106.

[32] C. H. Albright, A. Dueck, and W. Rodejohann, Eur. Phys. J. C 70, 1099 (2010).

[33] H. Ishimori, Y. Shimizu, M. Tanimoto, and A. Watanabe, Phys. Rev. D 83, 033004 (2011).

[34] X. G. He and A. Zee, Phys. Rev. D 84, 053004 (2011).

[35] S. F. King and C. Luhn, J. High Energy Phys. 09 (2011) 042.

[36] W. Rodejohann and H. Zhang, Phys. Rev. D 86, 093008 (2012).
[37] G. J. Ding, S. F. King, C. Luhn, and A. J. Stuart, J. High Energy Phys. 05 (2013) 084.

[38] C. Luhn, Nucl. Phys. B875, 80 (2013).

[39] Y. Shimizu, K. Takagi, and M. Tanimoto, J. High Energy Phys. 11 (2017) 201.

[40] I. Esteban, M. C. Gonzalez-Garcia, A. Hernandez-Cabezudo, M. Maltoni, and T. Schwetz, J. High Energy Phys. 01 (2019) 106.

[41] I. Esteban, M. C. Gonzalez-Garcia, A. Hernandez-Cabezudo, M. Maltoni, and T. Schwetz, NuFIT 4.0, http://www.nu-fit .org (2018).

[42] P. F. Harrison, D. H. Perkins, and W. G. Scott, Phys. Lett. B 530, 167 (2002).

[43] E. Ma and G. Rajasekaran, Phys. Rev. D 64, 113012 (2001).

[44] M. Tanabashi et al. (Particle Data Group), Phys. Rev. D 98, 030001 (2018).

[45] M. Dine, L. Randall, and S. D. Thomas, Phys. Rev. Lett. 75, 398 (1995).

[46] W. Buchmuller, K. Hamaguchi, O. Lebedev, and M. Ratz, Nucl. Phys. B699, 292 (2004).

[47] K. Nakayama and F. Takahashi, Phys. Lett. B 670, 434 (2009).

[48] B. Lillard, M. Ratz, T. M. P. Tait, and S. Trojanowski, J. Cosmol. Astropart. Phys. 07 (2018) 056.

[49] D. Hagihara, K. Hamaguchi, and K. Nakayama, J. Cosmol. Astropart. Phys. 03 (2019) 024.

[50] H. K. Dreiner, H. E. Haber, and S. P. Martin, Phys. Rep. 494, 1 (2010). 